\title{
Panorama general y reflexiones acerca de las relaciones entre Argentina y Corea
}

\author{
ㄴ. \\ Carolina Mera
}

Resumen

El presente artículo examina las relaciones entre la República Argentina y la República de Corea a lo largo de la historia, con el fin de focalizar su evolución reciente en el marco del proceso de globalización que ha transformado el mundo de las relaciones institucionales, políticas y económicas de las naciones. Desarrolla un panorama general del proceso por el cual actualmente América Latina se visualiza como posible socio estratégico y aplica políticas económico-comerciales, diplomáticas, culturales y académicas que le permiten acercarse a nuestro continente. El proceso se aborda como parte de la política interna y exterior actual de Corea, que busca estrechar relaciones con Argentina, el Mercosur y América Latina en general, como variable indispensable en esta etapa particular del desarrollo de su estructura económica y productiva y sobre todo, de un posicionamiento estratégico en el plano geopolítico.

Abstract

The present article analyzes the relationship between Argentina and the Republic of Korea throughout history, in order to focus recent trends in the framework of the globalization process that has transformed the world of political and economic institutional relationship within nations. It develops an overall view of the process by which, at the present time, Korea perceives Latin America as a potential strategic partner and it implements economic, commercial, diplomatic, cultural and academic policies allowing Korea to come close to our continent.

This process is approached as part of the present domestic and foreign Korean policy, which intends to foster relations with Argentina, Mercosur and Latin America in general, as a central aspect of this particular stage of its economic and productive structure of development, and particularly of its strategic geopolitical positioning. 


\section{INTRODUCCIÓN}

En el presente trabajo se analiza la evolución de las relaciones de Argentina y la República de Corea, a fin de identificar las tendencias recientes en el marco del proceso de globalización que ha transformado el mundo de las relaciones institucionales políticas y económicas de las naciones.

Se analiza el proceso por el cual actualmente Corea percibe a la Argentina y la región como un posible socio estratégico y aplica políticas económico-comerciales, diplomáticas, culturales y académicas que le permiten acercarse a nuestro continente.

El proceso se aborda como parte de una política nacional en que las relaciones con nuestro país, el Mercosur y América Latina son un elemento fundamental para el desarrollo de su estructura económica y productiva y sobre todo de un posicionamiento estratégico en el plano geopolítico. Se parte de la base de que el proceso de avance hacia acuerdos y tratados regionales conforma nuevo espacios económicos, políticos y culturales e impone lógicas que exigen mayor atención y análisis de parte de académicos, políticos y empresarios.
Evolución de las RELACIONES BILATERALES ENTRE ARGENTINA Y COREA

Pese a los criterios que aplican algunos académicos latinoamericanos para sistematizar la historia de las relaciones diplomáticas entre ambos países", podemos afirmar que la política argentina hacia Corea se caracterizó por planes coyunturales ligados a los gobiernos de turno y no por una política de planificación a mediano y largo plazos como en el caso de Corea del Sur.

Las relaciones formales entre Argentina y la República de Corea se iniciaron en los años sesenta. En febrero de 1962 culminó exitosamente una serie de conversaciones sostenidas por representantes de ambos países y días antes de su destitución, el Presidente Frondizi estableció relaciones diplomáticas con ese país. Al año siguiente fue designado el primer embajador coreano con jurisdicción en la República Argentina, Chung Il-kwon, embajador en Estados Unidos y concurrente en nuestro país. Corea del Sur abrió su embajada en Buenos Aires en diciembre de 1963 y envió como primer embajador de Corea del Sur en Buenos Aires a Bae Ui-hwan (Paz, 1996). El 18 de Junio de 1964 el presidente Arturo Illia creó la embajada de Argentina en Seúl, y nombró como pri-

\footnotetext{
1 Las relaciones bilaterales entre Corea Argentina se han clasificado según distintos períodos. El Prof. E. Oviedo (2003) propone hacerlo de acuerdo a si se mantienen relaciones con una o las dos Coreas, abarcando cuatro perfodos: el anterior a 1962; 1962-1973; 1973-1977; 1978-2006; en cambio el Prof. Gonzalo Paz (1996) realiza la división por décadas: $1960 ; 1970 ; 1980 ; 1990$, incluyendo en ellas las diferentes variables a analizar. En el presente trabajo nos acercamos más a la perspcctiva del Prof. Paz.
} 
mer embajador a Alejandro Galerce (Oviedo, 2003).

Durante la década de 1960, Argentina centró la atención en establecer vínculos formales bilaterales con la República de Corea, política condicionada por su posición en el mundo bipolar.

El gobierno de facto de Onganía (1966) fortaleció las relaciones con Corea del Sur en el marco de las llamadas «fronteras ideológicas». En 1968 se firmó el convenio cultural ${ }^{2}$ que creó una Comisión Mixta, compuesta de dos secciones, con sedes en Buenos Aires y Seúl. El 27 de marzo de 1968 se cerró la embajada argentina en Seúl, que se reabrió el 6 de octubre de 1969, con un encargado de negocios y bajo la jurisdicción de la Embajada en Japón (Oviedo, 2003). Lanusse mantuvo la política aplicada por Onganía y en agosto de 1972 se firmó el «Acuerdo por notas reversales para el Otorgamiento y la Protección de los Derechos de Patentes de Invención, Modelos de Utilidad, Diseños y Marcas de Fábrica a los Nacionales de Ambos Países».

El $1^{\circ}$ de junio de 1973 Cámpora estableció relaciones diplomáticas con Corea del Norte, estableciendo una política de doble reconocimiento que se mantendría durante el peronismo y el primer año de la dictadura, 1977. En julio de 1973 el gobiemo provisional de Lastiri abrió la embajada y la sección consular argentinas en Pyong Yang. Perón mantuvo esa orientación, y el 11 de diciembre de 1975 se firmó el Convenio comercial entre el Gobierno de la República Argentina y el Gobierno de la República Popular Democrática de Corea ${ }^{3}$. El artículo tercero del Convenio contemplaba la promoción de las visitas, el intercambio tecnológico de carácter comercial y el otorgamiento de facilidades a la otra parte para la celebración de exposiciones y el fomento del comercio en su territorio. Presentó cartas credenciales el embajador Guang Sung Zeng el 8 de abril de 1974. El 8 de agosto de 1975, con Isabel Perón en el poder, se firmó en Buenos Aires el Acta de la Comisión Mixta Cultural Argentino-Coreana y en noviembre del mismo año el Acta de la Comisión Mixta Cultural ArgentinoCoreana Sección Buenos Aires.

El golpe de Estado de marzo de 1976 trajo consigo una mejora de las relaciones entre Argentina y Corea del Sur y un empeoramiento de aquellas con Corea del Norte. El embajador norcoreano acreditado en Buenos Aires fue trasladado a Yugoslavia y la represen-

2 El convenio cultural fue firmado en Buenos Aires el 8 de agosto de 1968 por el ministro de Relaciones Exteriores y Culto, Nicanor Costa Ménde\%, y el embajador de la Repüblica de Corea en Argentina, Dong Sung Kim. Fue aprobado por Decreto Ley $N^{\circ} 18.387$, del 3 de octubre de 1969, y entró en vigor el 4 de enero de 1970.

3 El artículo III expresa que «Ambas partes se otorgarán reciprocamente el trato de la nación más favorecida, tanto en lo que concierne a tarifas aduaneras, derechos de toda clase, tasas, impuestos, reglamentaciones sobre circulación, transporte y distribución de mercaderías, como asimismo en lo que se refiere a licencias, reglamentos, formalidades, procedimientos y disposiciones vinculadas a la exportación e importación de mercaderías». 
tación quedó en manos de un encargado de negocios ad interim como jefe de Misión. El malestar en las relaciones se profundizó con el incidente generado por la quema de la embajada norcoreana en Buenos Aires, hecho que aceleró la ruptura de relaciones diplomáticas, implementada el 13 de junio de 1977 por decreto del dictador Rafael Videla.

Dado que el gobierno de Corea del Norte no había reconocido al régimen de facto que derrocó al gobierno peronista, con quien había promovido las relaciones políticas y económicas, se ordenó el abandono del territorio argentino de todos los integrantes de la representación diplomática de ese país acreditados en Buenos Aires. Una situación similar había ocurrido en Chile cuatro años antes ${ }^{4}$.

Desde entonces, Argentina solo ha mantenido relaciones diplomáticas con Corea del Sur. La última dictadura y los gobiernos constitucionales de Alfonsin, Menem, De la Rúa, Duhalde y la actual gestión de Kirchner mantienen relaciones bilaterales con Corea del Sur.

A partir de 1979 se produjo un cambio en la posición argentina respecto de Corea que se refleja en diversos foros internacionales. La estrategia de contención comunista desplegada por la República de Corea, Japón y los países de la ASEAN tuvo repercusiones en nuestro país y en 1979 se inauguraron la agregadurías militar, naval y aeronáu- tica, política que continúa con los gobiernos democráticos.

La década de los años ochenta se caracterizó por una relación bilateral de escaso intercambio. En mayo de 1981 se firmó el "Comunicado Conjunto entre el Ministro de Comercio e Industria de Corea y el Ministro de Comercio e Intereses Marítimos de la República Argentina" (ứnico instrumento firmado en la década), con el que se inicia una larga relación en materia pesquera entre ambos países, que representa parte importante de la relación comercial bilateral.

Años después, el ministro de relaciones exteriores de Corea del Sur, Lee Won-kyung, asistió a la ceremonia de asunción del mando del Presidente Alfonsín. En 1986 visitó Corea el ex presidente Arturo Frondizi. En la agenda bilateral siguieron predominando la energía nuclear, la pesca y la migración, esta última vinculada principalmente a proyectos agrícolas.

La década de 1990 fue escenario de un gran incremento del comercio y las inversiones, del intercambio de vistas diplomáticas y de la firma de tratados.

La política de Menem profundizó las relaciones bilaterales y multilaterales con el régimen de Seúl a partir de la realización de las primeras visitas diplomáticas: el presidente Kim Youngsan (1996), el vicepresidente de la Asamblea Nacional coreana, Kim JaeKwang (1991); el primer ministro

4 El gobierno de Salvador Allende estableció relaciones diplomáticas con Corea del Norte el $1^{\circ}$ de junio de 1972 . El gobierno de facto de Pinochet interrumpió las relaciones tres días después del golpe de Estado del 11 de septiembre de 1973. 
Chung Won-Shik (1992); el enviado especial Hong Soon-young (1994); el ex-primer ministro Hwang In-sung (1995), el ministro de relaciones exteriores Gong Ro-myung (1995) y el enviado especial Lee Hong-ku (1996). En el caso de Argentina, además del viaje presidencial (1995), se produjeron las visitas del canciller Di Tella (1991) y del ministro Cavallo (1994) y la firma de acuerdos sobre usos pacíficos de la energía nuclear; promoción y protección de inversiones y servicio de transporte aéreo. En agosto de 1995 se firmó el tratado de extradición y en septiembre del mismo año se firmó en Seúl el Memorandum de entendimiento sobre consultas periódicas de alto nivel.

La participación de nuestro país en la asamblea general de fundación de la Organización para el Desarrollo de la Energía en la Península Coreana, en 1995 (Korea Peninsula Development Energy Organization - KEDO), significó un avance importante para las relaciones entre ambos países ${ }^{s}$. La participación argentina en la KEDO se dió a través de la Comisión Nacional de Energía Atómica (CNEA), la que mediante un aporte económico simbólico tuvo un gran impacto político. Como miembro de la organización desde 1996, y de la Organización Internacional de Energía Atómica, el gobierno nacional manifestó su preocupación por la decisión del gobierno de Corea del Norte de inte- rrumpir el monitoreo multilateral de las actividades nucleares en ese país, y lo instó a cumplir sus obligaciones en materia nuclear. Para la Argentina, que desde su adhesión al tratado de TlateIolco en 1993 busca profundizar la cooperación en los usos pacíficos de la energía nuclear, el compromiso de desmantelar su programa nuclear por Corea del Norte que se presta para el incremento de la experiencia en un mercado altamente tecnológico y competitivo.

El Acuerdo sobre servicios aéreos fue firmado en Buenos Aires el 9 de septiembre de 1996. El 19 de marzo de 1999 se llevó a cabo la VI Reunión Plenaria del Comité Mixto Empresario Argentino, que tuvo como parte argentina a la Cámara Argentina de Comercio.

El primer ministro, Kim Yong-pil, asistió a la ceremonia de asunción del mando de Fernando De la Rúa. Durante su mandato De la Rúa continuó y amplió la cooperación en el ámbito científico y tecnológico y duplicó las exportaciones. El Acuerdo de cooperación científica y tecnológica fue firmado el 31 de octubre de 2000.

En el ámbito económico, las negociaciones del Mercosur con Corea ${ }^{6}$, a partir del primer encuentro de Asunción realizado en abril de 1997 , constituyen una forma indirecta de vincular a nuestro país con cl este asiático.

Además, la creación del Foro de

5 Fundada en marzo de 1995 por los gobiernos de Estados Unidos, Japón y Corea del Sur, se originó en el acuerdo firmado en diciembre de 1994 entre los Estados Únidos y Corea del Norte, en virtud del cual este último aceptó congelar y desmantelar su programa nuclear.

6 Brasil es el único país del Mercosur que reconoce a Corea del Norte, desde marzo de 2001. 
Cooperación América Latina-Asia del Este (FOCALAE) contribuye al mantenimiento de un diálogo directo y se suma al que ya se mantiene en Naciones Unidas y otros organismos multilaterales.

La política exterior argentina hacia la península continúa la cooperación y amistad con la República de Corea, como lo demuestra la reciente firma del convenio sobre pesca por la Secretaría de Agricultura, Ganadería, Pesca y Alimentación y el Ministerio de Asuntos Marítimos y de Pesca.

Durante la visita del presidente Roh Moo-hyun se firmó un Memorando de entendimiento entre el Banco de la Nación Argentina y el Banco de Importación y Exportación de Corea en virtud del cual el banco coreano otorgó un préstamo de 30 millones de dólares para financiar a importadores argentinos que quieran comprar productos coreanos. Si bien la cifra no es elevada, el gobierno argentino le atribuyó un importante valor simbólico porque fue el primer crédito que el recibió el país después de la declaración de cesación de pagos de la deuda externa, en diciembre de 2001.

Además, con motivo de esa visita, los dos gobiernos acordaron establecer una "Relación de cooperación amplia para la prosperidad común en el siglo
XXI», comprometerse con la no proliferación y el uso pacífico de la energía nuclear, trabajar juntos para la paz mundial y para hacer más representativo, democrático y eficiente el Consejo de Seguridad de las Naciones Unidas, fortalecer del sistema multilateral de comercio y promover la cooperación bilateral en ciencia, tecnología, cultura y educación?.

Desde 2005 Argentina y Corea coinciden en sus posturas en todos los organismos internacionales, en las elecciones de organismos internacionales tales como el Consejo de Seguridad de las Naciones Unidas y en el tema de la reforma de este ${ }^{8}$. Como principales miembros del UfC (Unidos por el Consenso) se han opuesto al G-4 (Japón, Alemania, Brasil y India). El gobierno coreano esperaba que la Argentina, en su calidad de miembro no permanente del Consejo, apoyara la candidatura del CanciIler coreano Ban Ki Moon, como Secretario General de las Naciones Unidas.

Además, Corea y Argentina mantienen un diálogo político de alto nivel, en la Consulta Política, acordada en 1995 que se reunió en tres oportunidades, en la Comisión Mixta de Asuntos Económicos, creada en el 1991 y en el Comité Conjunto para Asuntos Culturales, creado en 1968 .

7 "Joint Statement of the Presidents of the Republic of Korea and the Argentine Republic", Buenos Aires, 15 de noviembre de 2004. El texto original se pucde consultar en el sitio web <www.korea.net>.

8 "Los dos países han trabajado juntos durante más de 10 años como miembros del Coffee Club que se opone al incremento del número de micmbros permanentes» (Choo Jong Youn, 2006). 
ASPECTO DE INTERÉS DE LA POLÍTICA APLICADA POR COREA A ARgentina y América Latina

Según Kim (1998) en materia de orientación política respecto de América Latina, Corea siguió los lineamientos de los «tres pilares tradicionales» de su política exterior: ideología anticomunista, emigración internacional y limitadas relaciones comerciales ${ }^{9}$. De acuerdo con esto, sus lazos con nuestro país fueron débileș y se limitaron a ciertas políticas relacionadas con estos lineamientos: política interna de Corea, migraciones y relaciones comerciales.

Respecto del primer elemento que se relaciona con la ideología anticomunista, cabe mencionar que ella se debió a la situación interna de la península, que condicionó su política exterior. Recordemos que durante las décadas de los años sesenta, setenta y ochenta, la política exterior coreana se centró en los problemas de seguridad interna vinculados con la amenaza proveniente de Corea del Norte. Por su parte, en el plano interno se centró la lucha contra el comunismo mediante la construcción de un sistema político que a su vez sentaba las bases de una economía fuerte. No obstante que la política exterior buscó el apoyo de los líderes latinoamericanos al régimen surcoreano, podemos afirmar que la política hacia América Latina no era una prioridad para la cancillería coreana (Choe, 2006).

A finales de los años ochenta y en la década de 1990 las relaciones exteriores de Corea se basaron en una política democrática al interior del país y en una apertura al exterior. En cuanto a América Latina, el eje político buscó el apoyo de los líderes latinoamericanos para el acceso de Corea como miembro pleno en los principales foros internacionales. Esto significó cambios importantes en el área comercial y de inversión directa en Argentina y América Latina. (ver Bolinaga, 2005)

Cabe recordar que en 1991 ambas Coreas ingresaron simultáneamente en las Naciones Unidas, y que Corea del Sur estableció lazos diplomáticos con Rusia, China y los países ex satélites de la Unión Soviética. Además, hay que mencionar la política de Corea del Sur hacia Corea del Norte del presidente Kim Dae-jung, denominada "sunshine policy".

Según Bolinaga (2005), en 2001 y particularmente a partir del 11 de septiembre, se abrió una nueva etapa marcada por el regreso de los temas de seguridad internacional y regional, en que Corea del Sur busca el apoyo político de los países de América Latina contra el desarrollo armamentista de Corea del Norte.

A partir del gobierno de Roh se observan ciertos cambios de la política

- Kim Won-Ho, «Korean-Latin American Relations: Trend and Prospects", Korean Journal of Latin America Studies, Vol. 1 (1998), pp. 25-48. También en Kim Won-Ho, "Korea and Latin America: End of a Honeymoon?», Latin America in the International Financial Crisis, Edition $\mathrm{N}^{\circ}$ 56, mayo-agosto 1999. 
respecto de Argentina, que se caracterizan por el interés y promoción de los vínculos académicos, culturales y de intercambio informático y tecnológico. Esto sin duda es parte de una nueva política hacia el continente, pero también de una nueva forma de concebir a Corea en el mundo. Como sostiene el Ministro Choo Jong Youn, «el concepto de seguridad, al cual Corea le atribuía suma importancia, ha variado de 'seguridad nacional' a 'seguridad humana'». Es evidente que este desplazamiento involucra de manera muy particular los contactos socioculturales entre Corea y el mundo.

En cuanto a la emigración internacional, si bien las migraciones fueron un aspecto importante de las relaciones entre Corea y América Latina, ella constituyó parte del fenómeno de expulsión de Corea en que no se establecieron vínculos con las comunidades de la diáspora.

En Corea los primeros movimientos migratorios se iniciaron en 1962 con la "Ley 1030 de Migración al Extranjero", a partir de la cual América Latina se convirtió en destino privilegiado para los ciudadanos coreanos ${ }^{10}$.

Sin embargo, los primeros inmigrantes fueron militares norcoreanos que en 1956 y 1957 optaron por no volver a su país. Con el apoyo de organismos de las Naciones Unidas pasaron de Corea del Sur a la India y de allí alrededor de una docena de ellos se traslada- ron a Argentina y un grupo más numeroso a Brasil.

Unos años antes de la primera llegada oficial, en octubre de 1965, entraron al país por las fronteras terrestre algunos migrantes provenientes de Brasil, Bolivia y Paraguay.

Las corrientes migratorias, poco numerosas y asistemáticas, se fueron intensificándo en las décadas siguientes.

Aunque la intención era instalarse en colonias agrícolas, estas no prosperaron porque la mayoría de los migrantes nunca antes habían practicado actividades agrícolas ni rurales (a pesar de que era uno de los requisitos para la emigración) y porque se trataba de regiones poco desarrolladas, con infraestructura precaria y pocos servicios educativos y sanitarios. Por esta razón, terminaron movilizándose hacia las zonas urbanas de las ciudades próximas para dedicarse a actividades comerciales.

En abril de 1985 se firmó en Buenos Aires un Acta de procedimiento para el ingreso de inmigrantes coreanos a la Argentina. Entre 1984 y 1989 se otorgarán más de 11.000 permisos a familias. Y entre 1985 y 1989 llegó la mayor parte de esta población, provista de capital de inversión y con hábitos incorporados de una Corea moderna e industrializada.

Para la colectividad coreana la década de 1990 significó un crecimiento cualitativo y una disminución cuanti-

10 En 1962 se da una corriente migratoria de 30 mil coreanos hacia América Latina: a Brasil, Argentina, Paraguay y Bolivia (Kim Ill Soo, 1981; Lee Kyo Bom, 1992; Mera, Carolina, 2005). 
tativa. No hubo nuevas oleadas migratorias sino expulsión. Se dio un crecimiento económico importante en el área textil, en la pequeña $y$ mediana industria, y en el comercio mayorista y minorista. Esto implicó tanto el desarrollo institucional de las asociaciones e iglesias, como el aumento del número de locales de servicios y consumos étnicos ${ }^{n}$. Este proceso dejó su impronta en el espacio urbano, a través de marcas culturales y experiencias de vida particulares, que adoptan como punto de referencia la vida en Corea.

La crisis de 2001 tuvo su impacto en la comunidad coreana de Argentina que se reflejó en la expulsión de algunos y el empobrecimiento de otros. El número de inmigrantes provenientes de Corea del Sur alcanzó su punto máximo en 1990, cuando llegó a 40 mil personas, que se instalaron principalmente en la provincia y ciudad de Buenos Aires. En 1999 la cifra ascendía a 31 mil y solo bajó a 25 mil, y en el 2003 a 15 mil. En 2005 se constató un leve incremento de 19000 personas y actualmente fluctuaría en torno a las $20 \mathrm{mil}$ personas. Cabe destacar que la mayo- ría de los inmigrantes se dirigieron hacia ciertos países de América Latina como México y Brasil y una minoría de jóvenes profesionales se instalaría en los Estados Unidos, España o Corea ${ }^{12}$.

Finalmente, en lo que toca a las relaciones comerciales, si bien Argentina no fue un actor privilegiado, hubo algunos temas recurrentes como la pesca, y la cooperación en materia de energía atómica y eléctrica ${ }^{13}$.

En 1996, la visita del presidente Kim a Argentina fue un paso importante en la estrategia de diversificación económica y política de Corea. Se firmaron acuerdos tales como los de protección de inversiones, promoción y acuerdos pesqueros, orientados a proveer el marco institucional para la expansión del intercambio y la cooperación entre Corea y América Latina. Se acordó que había que reforzar la cooperación entre ambas regiones, mediante una participación más activa de las empresas coreanas en el desarrollo económico de la región (Kwak, 2003) ${ }^{14}$.

Se constató un acercamiento en el área económico-comercial. El volumen del intercambio comercial entre Corea

II Debemos recordar que el tipo de cambio 1 a 1 mantenido durante este período fue un elemento esencial para que este desarrollo pudiera darse.

12 Entre 1999 y 2001 abandonaron Argentina 6178 personas y de Paraguay 4222, mientras que en el mismo periodo Ilegaron a la región 8656 personas. Esto significa que un total de 19056 personas movilizándose, entre quienes ya residian en países de América Latina y los recién llegados. Más de! $90 \%$ de estas personas ingresarän a México (17121) y Brasil (1181), restando 754 que seguramente se dirigieron a Guatemala y Chile. (Datos de la Dirección de Migraciones del Ministerio de Relaciones Exteriores y Comercio de Corea, 2005).

13 En febrero de 1980 se firmó el Acuerdo de Cooperación Científica y Tecnológica entre la Comisión Nacional de Energía Atómica de Argentina y la Comisión de Energía Eléctrica de Corea.

:4 Citado por Choe Gyoung-Joo, 2006, "Política exterior de Corea hacia América Latina. Evolución, desafios y perspectivas futuras", It EECA, UNLP. 
y América Latina aumentó con tendencia a la complementariedad, en el sentido de que con su industria altamente desarrollada Corea aparece como capaz de transferir tecnología a los países de América Latina, mientras estos aparecen como proveedores de recursos naturales y materia prima ${ }^{15}$. Otro elemento que facilitó inserción de empresas coreanas en los países de América Latina fue la apertura $y$ liberalización de los principales países de la región, que dio lugar al incremento de las exportaciones y a la inversión directa de Corea en la región.

\section{UNA NUEVA ETAPA DE ENCUENTROS}

Sin duda los procesos de consolidación democrática y de globalización económica y cultural representan una nueva etapa de las relaciones entre Corea del Sur y Argentina después de los años noventa.

Los gobiernos democráticos imprimieron un nuevo aire a la política exterior de Corea. Recordemos que el primer presidente civil fue Kim Young Sam, en 1993 y lo siguieron en 1998 Kim Dae Jung y en 2003 Roh mun Hyon, el primero y el último de los cuales visitaron nuestro país. La gira pre- sidencial de Roh, en 2004, buscaba multiplicar y consolidar las instancias de cooperación con Brasil (su socio más importante en la región), Chile y Argentina, con quienes alcanzó el status de «Comprehensive Cooperative Relationship for the Common Prosperity in the 21st Century"-

Así, la política exterior de Corea comprende nuevos elementos articuladores relacionados con la consolidación del sistema político-institucional democrático; la nueva coyuntura internacional y la voluntad de Corea de entrar al mundo globalizado mediante una estrategia multilateral, y el desarrollo económico sostenido y la superación de las crisis financieras ${ }^{16}$.

Como veremos más adelante, estos tres elementos tendrán aplicación en Argentina y América Latina. Así, el lugar marginal que Argentina y la región de América Latina ocupaban en la escala de intereses de Corea va revirtiéndose gradual sostenidamente en la última década, especialmente a partir de la política de apertura al mundo del Presidente Kim Dae Jung. Como sostiene Choe (2006) «Este activo despliegue de la diplomacia, fue acompañado en el plano institucional por la creación del Departamento de Asuntos Latinoamericanos y del Caribe en la Cancillería coreana y del Consejo

is Para el caso de Argentina, ver Mara Barembaum (2005).

16 En ese sentido es pertinente retomar los cinco principios fundamentales de la nueva diplomacia coreana señalados por Kwak (2003): 1. globalización, 2. diversificación, 3 . multidimensión, 4. cooperación regional y 5 . la orientación futura de la política exterior" citado por Choe Gyoung-Joo, 2006, en «Política exterior de Corea hacia América Latina. Evolución, desafios y perspectivas futuras", II EECA, UNLP. 
Coreano de América Latina y el Caribe con sede en Seúl» ${ }^{17}$.

Ya hemos mencionado que desde mediados de los años $1990 \mathrm{y}$ específicamente de la década de 2000 , los lineamientos de la política coreana permiten hablar de una nueva etapa respecto de Argentina y América Latina. Como sostiene el Ministro Choo Jong Youn, "Corea ha venido importando desde hace mucho tiempo, recursos como el bronce y residuos de sojas exprimidos desde Argentina, pero la visita del presidente Roh Moo Hyun en Noviembre del 2004 llegó a ser una oportunidad para que Corea abriera sus ojos plenamente hacia los recursos naturales de la Argentina. Con la visita del presidente Roh, los dos países acordaron en impulsar una amplia cooperación bilateral en las áreas de agricultura, recursos petroliferos, forestación, pesca, minería y energía, de manera que, incluyendo el acuerdo de cooperacioin en el campo energético $y$ de minería entre el Ministerio de Industria y Recursos naturales de Corea y el Ministerio de Planificación Federal de Argentina, se han firmado en total 6 acuerdos relacionados con los recursos naturales entre los organismos concernientes a ambos países" (Choo Jong Youn, 2006). En junio de este año tuvo lugar en Buenos Aires la primera reunión de la Comisión Mixta en Energía y Recursos Naturales. Además, en la embajada de Corea en Argentina, se inauguró el Centro para la Cooperación coreano-sudamericana en energía y recursos naturales.

La inserción de Corea en el mundo globalizado se da a partir de una estrategia multilateral que prioriza su posicionamiento en los organismos internacionales y la celebración de acuerdos comerciales internacionales. Esta política se inició a fines de 1980 con motivo de las gestiones para obtener la sede de los Juegos Olímpicos y posteriormente del mundial de fútbol. Además, cabe mencionar el ingreso a organismos internacionales como las Naciones Unidas, la OMC y el FMI, para lo cual obtuvo el apoyo de Argentina.

En este sentido, la nueva política de inserción le ha dado buenos resultados al gobierno de Corea en América Latina. Al respecto cabe citar la entrada en vigencia del Tratado de Libre Comercio con Chile en 2004 (primer tratado de esta naturaleza firmado por Corea); el acceso de Argentina y Brasil para realizar un estudio de factibilidad para la suscripción de un acuerdo de comercio entre Corea y el Mercosur; la postura favorable de Argentina y Brasil al ingreso de Corea como miembro extraregional en el Banco Interamericano de Desarrollo; el apoyo de los tres países en torno a la solución pacífica del problema nuclear norcoreano y la firma de acuerdos de cooperación en materia de recursos naturales y energéticos, ciencia y tecnología.

Como sostiene Choe (2006) «En cuanto al desenvolvimiento de Corea en

17 Choe Gyoung-Joo (2006), «Política exterior de Corea hacia América Latina. Evolución, ibid. 
regímenes multilaterales que involucran a países de América Latina, observamos una activa diplomacia de su parte: Corea es observadora de la OEA (desde 1981) asî como también viene participando de las Reuniones de Secretarios del Grupo Río (desde 1995) y es miembro de FOCALAE, el foro que busca promover el diálogo político y económico así como cooperación en todas las áreas entre los países del este asiático y América Latina, siendo uno de los logros alcanzados más importantes, su ingreso al BID en calidad de miembro extrarregional" ${ }^{18}$.

Otras políticas adoptadas por Corea en función del proceso de desarrollo económico futuro se vinculan con el establecimiento de nuevas relaciones:

1. Creación de redes de intercambio en comunicación, ciencia y tecnología. El desarrollo económico sostenido y la superación de las crisis financieras sitúan al país en una nueva etapa de su desarrollo industrial y tecnológico. Corea tiene imperiosa necesidad de asegurar el acceso a recursos naturales y nuevas fuentes de energía.

Al respecto, el gobierno coreano tiene especialmente en cuenta el valor de América Latina como posible proveedora de recursos naturales, y procura aumentar su participación en empresas de la región para proyectos en los sectores energético y de recursos natura- les, así como en la compra por estas de alta tecnología de fabricación coreana.

2. Creación de redes en el ámbito académico. Por un lado, Corea cuenta con varias universidades, como la Seoul National University, Hankook, University of Foreign Studies, Pusan University y otras que a través de centros de estudios de América Latina fomentan la investigación sobre distintos temas relacionados con la región. Por el otro, en el ámbito académico de Argentina se realizó, con financiamiento de la Korea Foundation y el apoyo de la Embajada de Corea, se realizó el Primer Encuentro de Estudios Coreanos en América Latina, en el que participaron 30 académicos de 7 países del continente. El segundo se realizó en el Colegio de México en 2005 y el próximo está previsto para 2007, en Brasil. Se estableció el Centro de Estudios Corea-Argentina en la Universidad de Buenos Aires, el cual ya firmó convenios de cooperación con la Yonsei University y la Information, Communicaton and Thecnological University. Se firmó un acuerdo para crear un Centro de Estudios de Corea en la Universidad de San Pablo.

Además, se han creado otros Centros de Cooperación de Información y Tecnología en Brasil y Chile, y se fundó el Centro para la Cooperación Coreano-Sudamericana en Energía y Recursos Naturales, con sede en Buenos Aires.

18 Choe Gyoung-Joo (2006), Política exterior de Corea bacia América Latina. Evolución, desafios y perspectivas futuras, op. cit. 
3. Promoción y mejoramiento de la imagen de Corea. El ámbito cultural ocupa un lugar importante en la política internacional de Corea y por lo tanto tiene presencia en Argentina y América Latina. Se entiende hoy que para establecer vínculos hay que tener más conocimientos mutuos en diversas áreas. Así, como forma de mejorar las relaciones con Argentina y alcanzar los objetivos propuestos cada vez hay mayores oportuniclades de conocer producciones cinematográficas, bailes modernos $y$ tradicionales, $y$ exposiciones de artes plásticas. De hecho, para noviembre próximo está prevista la apertura en Buenos Aires del Centro Cultural Coreano Latinoamericano, cuya finalidad será difundir y promover la cultura coreana en América Latina.

4. Politica hacia las poblaciones coreanas. El nuevo planteamicuto de la política exterior del Estado $y$ su forma de posicionar a Corea en el mundo lleva a otorgarle un lugar prioritario a los coreanos que residen en el exterior. Cabe recordar que en América Latina actualmente hay unas $110 \mathrm{mil}$ personas de origen coreano. Debido a los conocimientos que ya tienen por su experiencia en América Latina, y porque todavía conservan el idioma y ciertos tra- zos culturales transmitidos por sus padres, se considera que son un gran capital que servirá de puente de comunicación y facilitará la llegada de las empresas coreanas a nuestros países y el conocimiento de las políticas del gobierno.

De hecho, la embajada permite conocer una serie de políticas del gobierno orientadas a organizar y promover las redes de residentes coreanos en el mundo, entre las comunidacies coreanas $y$ su país $y^{y}$ también entre ellas ${ }^{19}$. En este sentido, las relaciones con las comunidades de residentes coreanos en el extranjero se han vuelto prioridad, como políticas que construyen nexos y canales de comunicación entre los países y regiones. También se observa el interés de ciertos organismos gubernamentales por la situación de las comunidades de residentes coreanos.

Para crear las redes antes mencionadas se establecen relaciones diplomáticas bilaterales y multilaterales, pero además se han multiplicado los programas de financiamiento y cooperación de organismos de la cancillería y otros organismos tales como la Korea Foundation, la Korean Overseas Foundation, la Korea International Cooperation Agency (KOICA), KOTRA, KIEP, AKS, entre otros ${ }^{20}$.

19 «E! Gobierno corcano está organizando asambleas mundiales como la Convención Mundial de Empresarios Cortanos, a través de las instituciones como Fundación de Corca, Agencia Coreana para la Jromoción de Comercio e Inversión (KoTks), Asociación de Comercio Internacional de Corta (KrTA), para promover los encuentros de los empresarios coreanos en el exterior» (Choo Jong Youn, 2006).

Podemos mencionar los programas para estudiantes extranjeros organizados por la Fundación de Corea o el Ninisterio de Educación y Desarrollo de Recursos l-Jumanos cie Corea, y el programa de visita a Corea de hijos de residentes coreanos en la Argentina. 
Los cuatro aspectos mencionados apuntan a potenciar los recursos invertidos en América Latina y Argentina, mediante la planificación y desarrollo de una política más dinámica, canalizada a parcir de relaciones diplomáticas bilaterales con los países, de relaciones diplomáticas multilaterales en instancias institucionales que involucran a la región, como el Mercosur, el AJEC o el FOCALAE (Foro de Cooperación Latinoamericano- Asia del Este), la ZICOSUR (Zona de integración del centro oeste de América del Sur), orientadas a entablar diálogos estables y de intercambio mutuo entre Corea y los países de la región.

\section{Conclusiones}

El incremento del intercambio económico, cultural y científico entre Corea y Argentina se debe a una política planificada de Corea hacia nuestro continente. América Latina ofrece una complementariedad óptima en la actual etapa de la política de Corea.

Por un lado, América Latina es percibicla como proveedora de recursos naturales y energéticos por lo cual se prevé que empresas coreanas realizarán inversiones en el proceso de explotación; y por el otro, como potencial socio comercial de grandes empresas coreanas, tales como LG, Hyundai, Samsung, KIA, que podrían instalar sus fábricas $y$ entrar competitivamente al mercado interno. Para alcanzar estos objetivos, se han desplegado recursos diplomáticos, culturales, académicos y financieros.

La situación revela que enfrentamos un fuerte desafío. América Latina se encuentra en una coyuntura óptima para cntablar nuevas rclaciones con Corea (y los países de Asia) que a largo y corto plazo influyan favorablemente en nuestras economías. Sin embargo, para esto es importante pensar en un modelo de desarrollo que trascienda el intercambio de materias primas y alimentos por productos con valor agregado. El este de Asia adquirió mayor relevancia mediática a partir del potencia! económico que representa para nuestras economías. El tema pasa por promover relaciones con socios económicos que nos permitirian entrar en un mercado competitivo de alimentos y materias primas, a la región del mundo que compra un mayor volumen de estos productos.

Al respecto, hay que destacar el potencial del ámbito académico, pese a que en este aspecto los estudios realizados en los países de América Latina se limitan a estudiar la lógica de los procesos económicos, o la política de seguridad internacional a partir del conflicto con Corea del Norte. En cambio, no se presta cspecial interés, por ejemplo, a la experiencia de Corea en relación con el desarrollo económico, el crecimiento $y$ la inversión en educación, ciencia y tecnología.

La política exterior de Corea contiene innovaciones respecto de sus áreas de involucramiento, basadas en la etapa actual de su crecimiento.

Cabe preguntarse cómo se posiciona América Latina en este diálogo. Hay 
dos perfiles de respuesta: seguir la orientación de Corea e insertarse como productora de materias primas y recursos naturales y energéticos, o incorporarse a la negociación a partir de un proyecto de desarrollo conjunto.

Podríamos pensar que al igual que lo hace Corea, los países de América Latina deberían poner en ejecución planes de intercambio cultural $y$ académico. Existe una barrera cultural que en muchos sentidos impide avanzar en el contacto político, económico y' social con las naciones el este asiático. Para comprender la región hay' que derribar mitos, prejuicios y estercotipos que distorsionan el entendimiento general del escenario actual. Los intercambios desde Argentina y América Latina siguen estando dominados por la posibilidad de obtener utilidades económicas. $y$ esto se percibe en el ámbito académico latinoamericano, donde la mayoría de los investigadores se concentran en el área de economía internacional. Todavía no se valoran esos elementos que, como hemos observado, Corea ya visualizó de manera particular para esta etapa de su desarrollo global.

En este punto, debemos mencionar que también las comunidades coreanas residentes en nuestro continentc fueron concebidas como agentes dinámicos que forman parte del creciente intercambio comercial en distintas etapas de las relaciones bilaterales. Sin embargo, no se supo aprovechar estos recursos humanos como actores culturales anclados en nuestras propias realidades locales, con un potencial de transmisión de la lengua $y^{\prime}$ un acervo cultural imprescindible para el conocimiento mutuo.

Finalmente, hay que analizar la relevancia adquirida por China en el sistema internacional, ya que significa un impacto no solo para occidente sino también para otros países de la región, como Corea, Japón y el sudeste asiático, que han debido adaptar y modificar sus economías y políticas internacionales frente a las estrategias de la apertura de China.

\section{Bibliografía}

Barembaum, Mara (2005), "Complementariedad agro-industria! entre Argentina y la república de Corea del Surn, Tesina de la Carrera de Economía Agraria, Facultad de Agronomía, UBA.

Bauman, Zygmunt (2000), Modernidad liquida, ICCE, Buenos Aires; Argentina.

Bolinaga, Luciano (2005), "La diplomacia coreana en América Latina a principios del Siglo xxl: cambio y continuidad de su modelo de vinculaciốn». Disponible en www.ceaa.colmex.mx/estudioscoreanos/ images/damian.pdf

Choe Gyoung-Joo (2006), "Política exterior de Corea lacia América Latina. Evolución, desafíos y perspectivas futuras", ponencia presentada en el Segundo Encuentro de Estudios Corcanos en Argentina, Universidad de La Plata, Argentina. Choo Jong Youn (2006), «Un nuevo enfoque de la relación Corea-Argentina", trabajo presentado en el Segundo Encuentro de Estudios Coreanos en Argentina, Universidacl de Lal Plata, Argentina.

Di Masi, Jorge (2005), «Relaciones preferenciales Mercosur-Coren: perspectivas cle una idea en construcción". Disponible en: www.ccaa.colmex.mx/estudioscorcanos/images 
Girado Gustavo (2003), Comercio Argentina I Asia Pacífico. Una carrera de obstáculos, Corregidor, Bucnos Aires, Argentina.

Kim, Won Ho (1998), “Korcan-Latin American Relations: Trends and Prospects,» Korean Journal of Latin American Stuclies Vol. 1.

Kwak, Jae-Sung (2003), "Relaciones entre Corea $y$ América Latina en el Siglo XXI: una perspectiva coreana", en Señas de identidad coreana, Ojeda /Hidalgo/de Laurentis (coordinadores), Centro Espanol de Investigaciones Coreanas, Ed. Gondo, S.A. Toledo, España.

Mera, Carolina (1998), La inmigración coreana en Buenos Aires. Multiculturalismo en el espacio urbano, Eudeba, Buenos Aires, Argentina.

Mera, Carolina (2003), Estudios Coreanos en América Latina, Ed. Al margen, La l'lata, Argentina.

Mera, Carolina (2005), «Diásporn coreana en América Latina», II Encuentro de Estu- dios Coreanos en América Latina, Colegio de México- Korea Foundation. Disponible en: http://cena.colmex.mx/ ustudioscorcanos/images/mera.pdf

Oviedo, Eduardo (2005), "La política exterior de Argentina hacia la península de Corea en el ámbito multilateral", en Mera Carolina (comp.) Encuentro de Estudios Coreanos en América Latina, Ed. Al margen, La Plata, Argentina

Página oficial de FOCALAE - Corea, <http:// fealac.mofat.go.kr/span/information/ brief.php>.

Paz, Gonzalo (1996), "Las relaciones entre Corea y la Argentima. Desarrollo y perspectivas", Disponible en http://wwwasiayargentina.com/cari-13.htm

<www.korea.nets.

Estadísticas de la Dirección de Migraciones del Ministcrio de Relaciones Extcriores y Comercio de Corea (2005), wiww.mofat.go.kr 